\title{
Role of heavier-than-proton nuclei in neutron monitor response
}

\author{
Sergey Koldobskiy, ${ }^{a, b, *}$ Gennady Kovaltsov ${ }^{c}$ and Ilya Usoskin ${ }^{a}$ \\ ${ }^{a}$ University of Oulu, Finland \\ ${ }^{b}$ National Research Nuclear University MEPhI, Moscow, Russia \\ ${ }^{c}$ Ioffe Physical-Technical Institute, St. Petersburg, Russia \\ E-mail: sergey.koldobskiy@oulu.fi
}

Heavier-than-proton nuclei in cosmic rays are responsible for up to $45 \%$ of neutron monitor (NM) response depending on the level of solar modulation as well as the geomagnetic rigidity cutoff and altitude of a given NM location. Therefore, careful consideration of these heavy species is important for an accurate analysis of NM data, including a reconstruction of the solar modulation potential using the worldwide NM network data. Recently, the AMS-02 experiment allowed us to directly verify the NM response to heavy particles. In this work, we evaluate the expected contribution of heavy nuclei into the NM response considering different models of the local interstellar spectrum and also for different levels of solar activity.

$37^{\text {th }}$ International Cosmic Ray Conference (ICRC 2021)

July 12th - 23rd, 2021

Online - Berlin, Germany

\footnotetext{
${ }^{*}$ Presenter
} 


\section{Introduction}

Neutron monitors (NMs) are the main detectors aimed to study the long-term cosmic-ray variability, thanks to the long period ( $\sim 70$ years) of their operation. NMs register mainly the nucleon component of secondary particle showers, produced during interactions of primary cosmic rays with the nuclei of the atmosphere. NMs are typically register secondaries produced by Galactic cosmic rays (GCRs), but sometimes they can also observe secondary particles produced by high-energy solar energetic particles (SEPs), events of their observation are rare (72 events from 1956 until today) and called ground level enhancements (GLE, the GLE data is available at the International GLE Database (IGLED) [1] on https://gle . oulu . fi).

The most appropriate way of the NM analysis is the use of the NM yield function (YF) which allows to calculate the expected NM response $N_{\text {th }}$ knowing the flux of the primary particles $J$ :

$$
N_{\mathrm{th}}=\sum_{i} \int_{P_{c}}^{\infty} J_{i}(E) Y_{i}(E) d E
$$

where the summation is over the number of considered cosmic-ray species, and $P_{c}$ is the cutoff rigidity of a given NM location. Recently, different yield functions were tested against cosmic-ray fluxes measured in space [2] and the best performance was shown by YF calculated by Mishev et al. [3], which was recently expanded to different atmospheric depths [4].

The nucleon component of GCRs is composed of protons (about $90 \%$ in the number of particles), helium nuclei $(\simeq 8 \%$ ), and heavier-than-helium nuclei, which can be effectively represented by helium nuclei. In paper [2], careful consideration of heavy nuclei was performed on the basis of AMS-02 measurements [5]. The heliospheric modulation [6] of heavier-than-helium CR species in terms of rigidity is expected to be similar to that of helium nuclei since these heavy nuclei have almost the same charge-to-mass ratio, $Z / A \approx 0.5$, so that the ratio of heavy elements to helium was calculated and used for consideration of heavy nuclei.

The direct comparison between the fluxes of CR observed in space and NM responses is available only for the last $\approx 15$ years, when the PAMELA [7] and AMS-02 experiments were/are in operation, If one wants to analyze the cosmic-ray variability on the long-term scale, the modeled local interstellar spectrum (LIS) together with some model of GCR modulation inside the heliosphere should be used, typically the simplified one-parameter model of solar modulation [8] is used since within this model the solar modulation can be described with only one parameter. It was shown [2] that the LIS by Vos and Potgieter [9] can describe the observed cosmic-ray proton flux very well and that the helium nuclei can be effectively represented in this approach as an additional coefficient dependent on the solar modulation potential $\phi$ (on average, this coefficient is 0.353 ).

In this work, we will quantify the role of heavy nuclei in the NM response using recent AMS-02 observations and different LIS models.

\section{Quantifying the heavy nuclei response}

In this study, the AMS-02 experiment data on proton and helium CR fluxes variability [5] was used. The data with Bartels Rotation (BR, 27-day) cadence is available for the period 2011-2017 and covers the period of increasing solar activity, solar maximum, and decreasing solar activity. In 


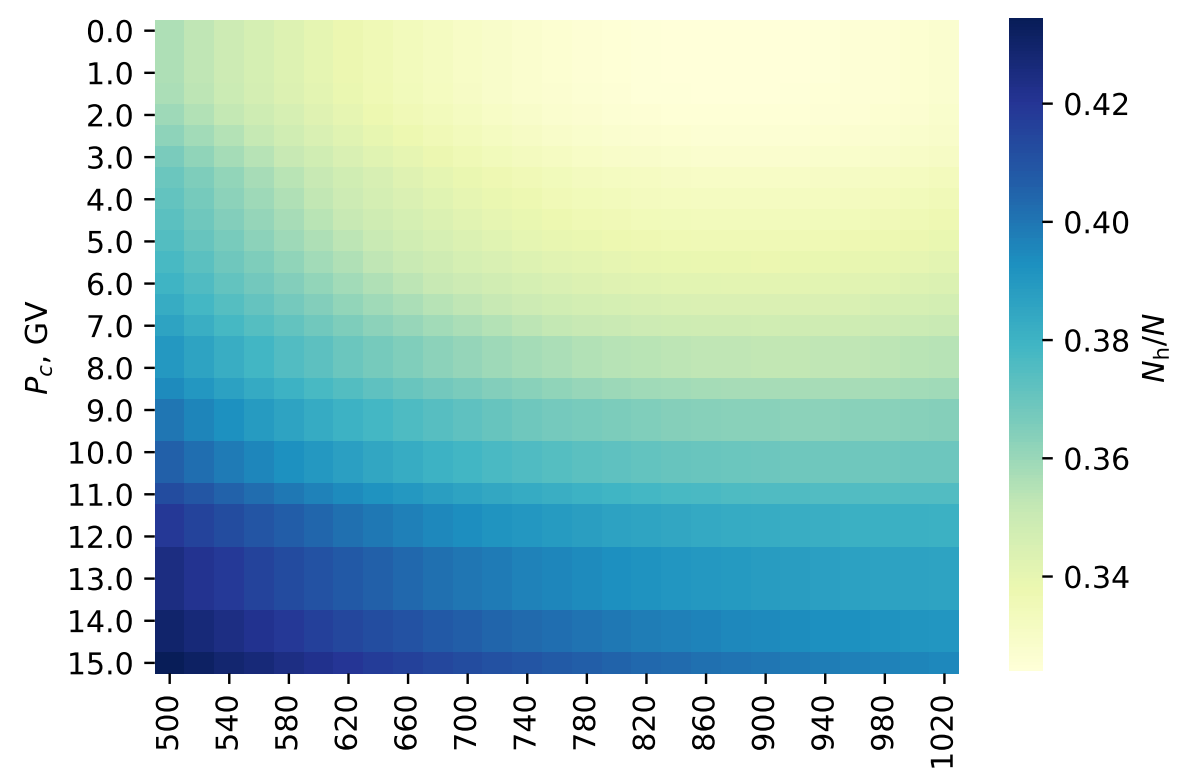

Atmospheric depth, $\mathrm{g} / \mathrm{cm}^{2}$

Figure 1: The relative contribution of heavy nuclei into the NM response as a function of cutoff rigidity $P_{c}$ and atmospheric depth as obtained using the AMS-02 experimental data for BR 2462.

Fig. 1 we show the computed ratio $N_{\mathrm{h}} / N$ between the contribution to NM count rate from heavy particles (helium and heavier nuclei) $N_{\mathrm{h}}$ and the full NM count rate, $N$, computed for an ideal NM64-type NM for a wide range of atmospheric depths and cutoff rigidities using Eq. 1 and NM YF from [4]. Here the AMS-02 data for the maximum of solar activity (BR 2462, 11-Jan-2014 07-Feb-2014) was used. One can see that the ratio $N_{\mathrm{h}} / N$ rises with the cutoff rigidity and decreases with the atmospheric depth, being about 0.33 for a polar sea-level NM and $\sim 0.43$ for a high-altitude equatorial NM. We also note that the main part of active NMs is located in mid- and high latitudes and only a small number of NMs are located close to the equatorial zone, where the big values of cutoff rigidities are observed.

The solar cycle dependence of $N_{\mathrm{h}} / N$ is shown in Fig. 2 for a polar sea-level NM $\left(P_{c}=0.1 \mathrm{GV}\right.$, $d=1033 \mathrm{~g} / \mathrm{cm}^{2}$ ). This dependence is less prominent in comparison to location-depended changes, being of the order of $3 \%$ for the period of AMS- 02 observations for polar sea-level NMs which are more sensitive to the solar cycle than low-latitude NMs.

The use of AMS-02 data, therefore, allows us to directly quantify the contribution of heavy nuclei species to the NM response. Taking into account the fact that solar modulation of protons and helium nuclei is charge-, time- and energy-dependent as obtained by AMS-02 and PAMELA experiments $[10,11]$ and another fact that neutron monitors are energy-integrating devices, we are currently unable to reconstruct proton and helium fluxes separately from NM data.

However, if one wants to use the NM data for the study of solar-cycle induced variation of GCR fluxes, qualitative and quantitative consideration of heavy nuclei should be done. Here we emphasize that the approach of solar modulation potential (force-field model) is typically used for the description of long-term variations. In the framework of this approach solar modulation and 


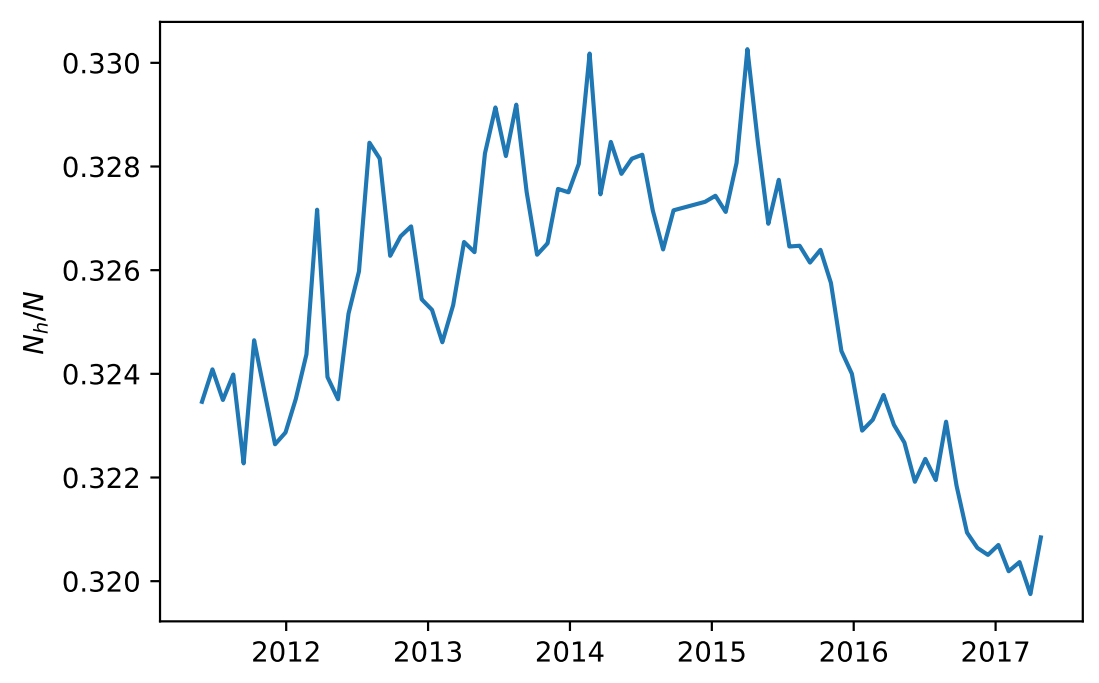

Figure 2: Contribution of heavy nuclei into the polar sea-level $\left(P_{c}=0.1 \mathrm{GV}, d=1033 \mathrm{~g} / \mathrm{cm}^{2}\right) \mathrm{NM}$ response as a function of time obtained using direct observational data from the AMS-02 experiment.

the corresponding GCR variability are described with only one parameter, the solar modulation potential $\phi$. The corresponding modulation of CR fluxes can be described using the simple analytical expression. Modeled LIS are typically used together with force-field model to describe CR fluxes observed near Earth.

Previously, in the framework of this approach, the constant coefficient of 0.3 was used to account for heavy nuclei, this coefficient was multiplied by the proton flux and NM YF for $\alpha$-particles using Eq. 1. However, the AMS-02 data allow one to calculate this coefficient directly. In order to do it, the response of a standard NM due to $(Z>1)$ GCR species, $N_{\mathrm{h}, \mathrm{AMS}}$, to the corresponding response, $N_{\mathrm{h} \text {,mod }}$, calculated by applying the standard force-field approach to the proton LIS, as a function of the modulation potential $\phi$, namely, $C(\phi)=N_{\mathrm{h}, \mathrm{AMS}}(\phi) / N_{\mathrm{h}, \bmod }(\phi)$ was calculated [2].

First, we tested the LIS of Vos \& Potgieter [9], constructed using the PAMELA proton observations for period 2006-2009 [12], Voyager observations beyond heliosphere [13], GALPRPOP model $[14,15]$ for cosmic-ray propagation in the Galaxy and numerical simulations of cosmic-ray solar modulation by Potgieter et al. Using this LIS, we obtained the mean value of $C=0.353$ and also we evaluated $C$ as a function of the solar modulation potential $\phi$ (Figure 3), so that the clear tendency of increasing $C$ with the increase of $\phi$ value was obtained.

Here we also test the recent LIS estimation by Boschini et al. [16], obtained using AMS-02 and Voyager experimental data together with GALPROP and with the use of numerical simulation of cosmic-ray solar modulation using HelMod code [17]. Testing this estimation together with the AMS-02 data using the force-field approach allows us to conclude that it gives almost the same qualitative description of the solar modulation effects that differ in the value of the obtained solar modulation potential $\phi$ with the mean difference being about $20 \mathrm{MV}$. At the same time, the LIS model by Boschini et al. 2020 allows to get significantly lower values of the $\chi^{2}$ during the best-fit 


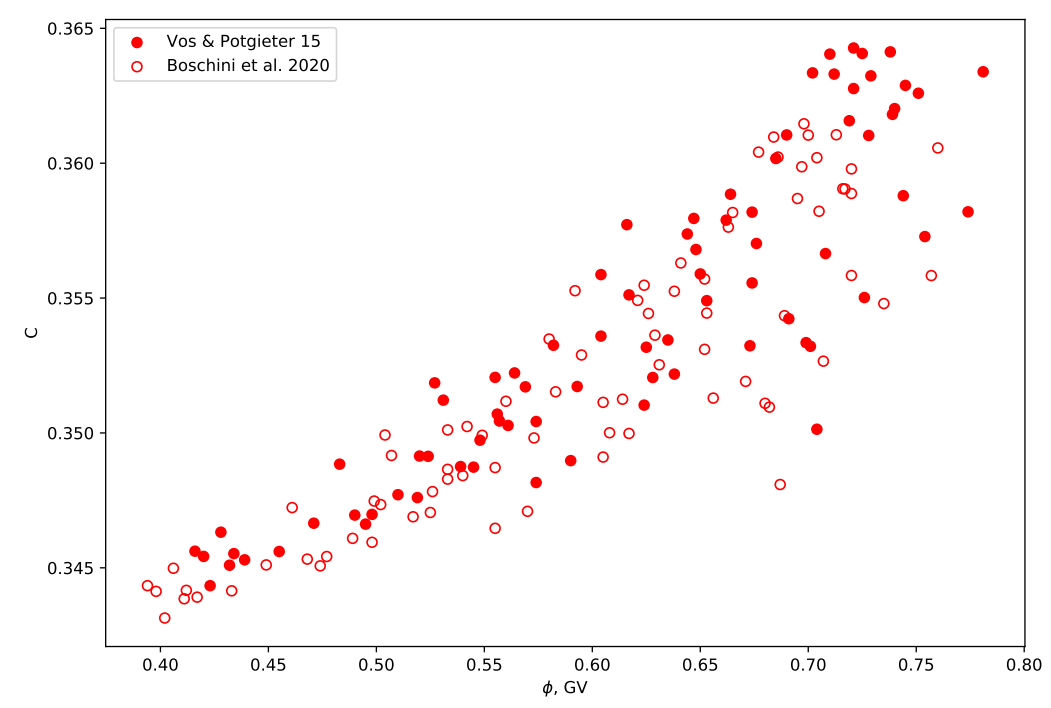

Figure 3: Dependence of the ratio $C=N_{\mathrm{h}, \mathrm{AMS}}(\phi) / N_{\mathrm{h} \text {, mod }}$ on the modulation potential $\phi$ considering two LIS models: Vos \& Potgieter 2015 [9] (full dots) and Boschini et al. 2020 [16] (empty dots).

finding procedure, that means that this LIS is more capable for the description of the observed spectra.

Evidently, both models give similar quantitative and qualitative description of the $N_{\mathrm{h}, \mathrm{AMS}}(\phi) / N_{\mathrm{h}, \bmod }(\phi)$ ratio.

\section{Conclusion}

In this work we compare the expected contribution of heavy nuclei into the NM response using AMS-02 experimental data and recent estimation of NM YF [4]. We found that heavy nuclei can be responsible for up to $\sim 43 \%$ of NM counts depending on the location of NM (namely, its cutoff rigidity and altitude). Solar-cycle dependence of the ratio $N_{h} / N$ (between the heavy-nuclei-induced NM signal and full NM signal) is less prominent in comparison to location-dependent changes, being about $3 \%$ for the period of AMS-02 observations.

We also tested how the use of different LIS can change the heavy nuclei signal in full NM count rate in the framework of a simple force-field model of the GCR solar modulation. We found that the use of LIS by Vos \& Potgieter, 2015 [9] and Boschini et al. 2020 [16] gives the same dependence of the ratio $C=N_{\mathrm{h}, \mathrm{AMS}}(\phi) / N_{\mathrm{h} \text {, mod }}$ on the solar modulation potential.

Obtained results allow us to better understand the role of heavy nuclei in the neutron monitor response and also carefully quantify it.

\section{Acknowledgments}

This work was partially supported by the Academy of Finland (projects No. 321882 ESPERA, 330063 QUASARE). S.K. acknowledges support by the Russian Science Foundation project no. 20-72-10170. 


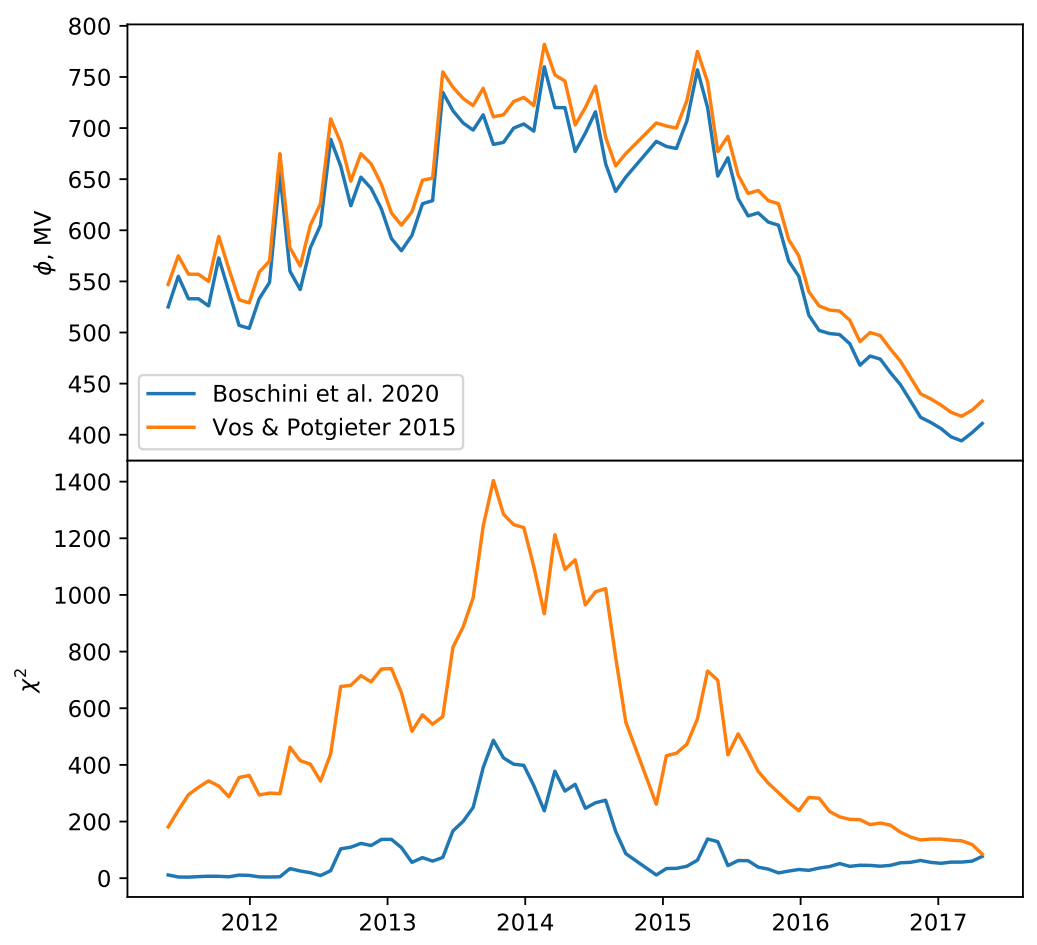

Figure 4: Solar modulation potential $\phi$ (upper panel) and the corresponding quality of the best fit $\chi^{2}$ (lower panel) as obtained for AMS-02 data using two LIS models: Vos \& Potgieter 2015 [9] (orange line) and Boschini et al. 2020 [16] (blue line).

\section{References}

[1] I. Usoskin, S. Koldobskiy, G.A. Kovaltsov, A. Gil, I. Usoskina, T. Willamo et al., Revised GLE database: Fluences of solar energetic particles as measured by the neutron-monitor network since 1956, Astronomy \& Astrophysics 640 (2020) A17.

[2] S.A. Koldobskiy, V. Bindi, C. Corti, G.A. Kovaltsov and I.G. Usoskin, Validation of the neutron monitor yield function using data from AMS-02 experiment, 2011-2017, Journal of Geophysical Research: Space Physics 124 (2019) 2367 [1904 . 01929].

[3] A.L. Mishev, I.G. Usoskin and G.A. Kovaltsov, Neutron monitor yield function: New improved computations, Journal of Geophysical Research: Space Physics 118 (2013) 2783.

[4] A.L. Mishev, S.A. Koldobskiy, G.A. Kovaltsov, A. Gil and I.G. Usoskin, Updated Neutron-Monitor Yield Function: Bridging Between In Situ and Ground-Based Cosmic Ray Measurements, Journal of Geophysical Research: Space Physics 125 (2020) e2019JA027433.

[5] M. Aguilar, L. Ali Cavasonza, B. Alpat, G. Ambrosi, L. Arruda, N. Attig et al., Observation of Fine Time Structures in the Cosmic Proton and Helium Fluxes with the Alpha Magnetic Spectrometer on the International Space Station, Physical Review Letters 121 (2018) 051101. 
[6] M. Potgieter, Solar Modulation of Cosmic Rays, Living Reviews in Solar Physics 10 (2013) 3.

[7] O. Adriani, G.C. Barbarino, G.A. Bazilevskaya, R. Bellotti, M. Boezio, E.A. Bogomolov et al., Ten Years of PAMELA in Space, Rivista del Nuovo Cimento 40 (2017) 473 [1801. 10310].

[8] L.J. Gleeson and W.I. Axford, Solar Modulation of Galactic Cosmic Rays, The Astrophysical Journal 154 (1968) 1011.

[9] E.E. Vos and M.S. Potgieter, New modeling of galactic proton modulation during the minimum of solar cycle 23/24, The Astrophysical Journal 815 (2015) 119.

[10] O. Adriani, G.C. Barbarino, G.A. Bazilevskaya, R. Bellotti, M. Boezio, E.A. Bogomolov et al., Time dependence of the proton flux measured by PAMELA during the July 2006 December 2009 solar minimum, The Astrophysical Journal 765 (2013) 11 [1301. 4108].

[11] N. Marcelli, M. Boezio, A. Lenni, W. Menn, R. Munini, O.P.M. Aslam et al., Time Dependence of the Flux of Helium Nuclei in Cosmic Rays Measured by the PAMELA Experiment between 2006 July and 2009 December, The Astrophysical Journal 893 (2020) 145.

[12] O. Adriani, G.C. Barbarino, G.A. Bazilevskaya, R. Bellotti, M. Boezio, E.A. Bogomolov et al., Measurement of the isotopic composition of hydrogen and helium nuclei in cosmic rays with the PAMELA experiment, The Astrophysical Journal 770 (2013) 2 [1304 . 5420].

[13] E.C. Stone, A.C. Cummings, F.B. McDonald, B.C. Heikkila, N. Lal and W.R. Webber, Voyager 1 observes low-energy galactic cosmic rays in a region depleted of heliospheric ions, Science 341 (2013) 150.

[14] I.V. Moskalenko and A.W. Strong, Production and Propagation of Cosmic-Ray Positrons and Electrons, The Astrophysical Journal 493 (1998) 694.

[15] A.W. Strong and I.V. Moskalenko, Propagation of Cosmic-Ray Nucleons in the Galaxy, The Astrophysical Journal 509 (1998) 212 [9807150].

[16] M.J. Boschini, S.D. Torre, M. Gervasi, D. Grandi, G. Jóhannesson, G.L. Vacca et al., Inference of the Local Interstellar Spectra of Cosmic-Ray Nuclei $Z \leq 28$ with the GalProp-HelMod Framework, The Astrophysical Journal Supplement Series 250 (2020) 27 [2006.01337].

[17] M.J. Boschini, S. Della Torre, M. Gervasi, G. La Vacca and P.G. Rancoita, The HELMOD model in the works for inner and outer heliosphere: From AMS to Voyager probes observations, Advances in Space Research 64 (2019) 2459 [1903 . 07501]. 